\title{
Preference Graph of Potential Method as a Fuzzy Graph
}

\author{
Siti Salwana Mamat, ${ }^{1}$ Siti Rahmah Awang, ${ }^{2}$ and Tahir Ahmad $\mathbb{D}^{1,3}$ \\ ${ }^{1}$ Department of Mathematical Sciences, Faculty of Science, Universiti Teknologi Malaysia, 81310 Johor Bahru, Malaysia \\ ${ }^{2}$ Azman Hashim International Business School, Universiti Teknologi Malaysia, 81310 Johor Bahru, Malaysia \\ ${ }^{3}$ Centre of Sustainable Nanomaterials, Ibnu Sina Institute for Scientific and Industrial Research, Universiti Teknologi Malaysia, \\ 81310 Johor Bahru, Malaysia
}

Correspondence should be addressed to Tahir Ahmad; tahir@ibnusina.utm.my

Received 21 August 2019; Revised 4 December 2019; Accepted 27 December 2019; Published 19 February 2020

Academic Editor: Zeki Ayag

Copyright (c) 2020 Siti Salwana Mamat et al. This is an open access article distributed under the Creative Commons Attribution License, which permits unrestricted use, distribution, and reproduction in any medium, provided the original work is properly cited.

\begin{abstract}
An autocatalytic set (ACS) is a graph. On the other hand, the Potential Method (PM) is an established graph based concept for optimization purpose. Firstly, a restricted form of ACS, namely, weak autocatalytic set (WACS), a derivation of transitive tournament, is introduced in this study. Then, a new mathematical concept, namely, fuzzy weak autocatalytic set (FWACS), is defined and its relations to transitive PM are established. Some theorems are proven to highlight their relations. Finally, this paper concludes that any preference graph is a fuzzy graph Type 5.
\end{abstract}

\section{Introduction}

Graph theory is rapidly moving into diverse fields such as chemistry, engineering, computer science, and operations research [1-3]. Graph has been used to model interconnections between natural and man-made systems. Dey and Pal [4] and Dey et al. [5] used graph-coloring technique to classify traffic accidental zones. Dey et al. [6, 7] solved fuzzy shortest path problems with interval Type 2 fuzzy arc lengths of a graph. Wuest et al. [8] utilized a graph to determine quality of a manufacturing program.

Two structures of interest in this study are ACS and PM where both are graphs. Each one of them contains a set of vertices and a set of edges. However, the ACS is a graph such that each vertex has at least an incoming link, whereas the $\mathrm{PM}$, on the contrary, must always have a vertex with a missing incoming link. The relation between these two mathematical structures has not been explored yet. Therefore, the anticipated relation is studied and presented in this paper.

Firstly, a weak form of ACS, namely, weak autocatalytic set (WACS), is introduced to serve as a "bridge" between ACS and PM. The study is later expanded to the case of PM with uncertainty connections between its vertices, i.e., fuzzy edges. The idea has led to a new ACS, namely, fuzzy weak autocatalytic set (FWACS).

The paper is organized as follows. Section 2 provides some basic concepts and definitions associated with this study. The WACS is introduced in Section 3 and some features of WACS are presented in this section. Section 4 elaborates ACS and PM and their relation. The fuzzification of WACS is described in Section 5. Finally, the conclusion of this paper is presented in Section 6.

\section{Preliminaries}

Generally, a graph represents a relationship between objects visually. These objects are represented by vertices and their relations by edges (see Figure 1).

The formal definition of a graph is given in Definition 1. An example of a graph with four vertices $V=\left\{v_{1}, v_{2}, v_{3}, v_{4}\right\}$ is illustrated in Figure 2.

Definition 1 (see [10]). A graph is a pair of sets $(V, E)$ where $V$ is the set of vertices and $E$ is the set of edges.

Furthermore, another way to represent a graph is by its adjacency matrix. The definition of an adjacency matrix for a given graph is as follows. 


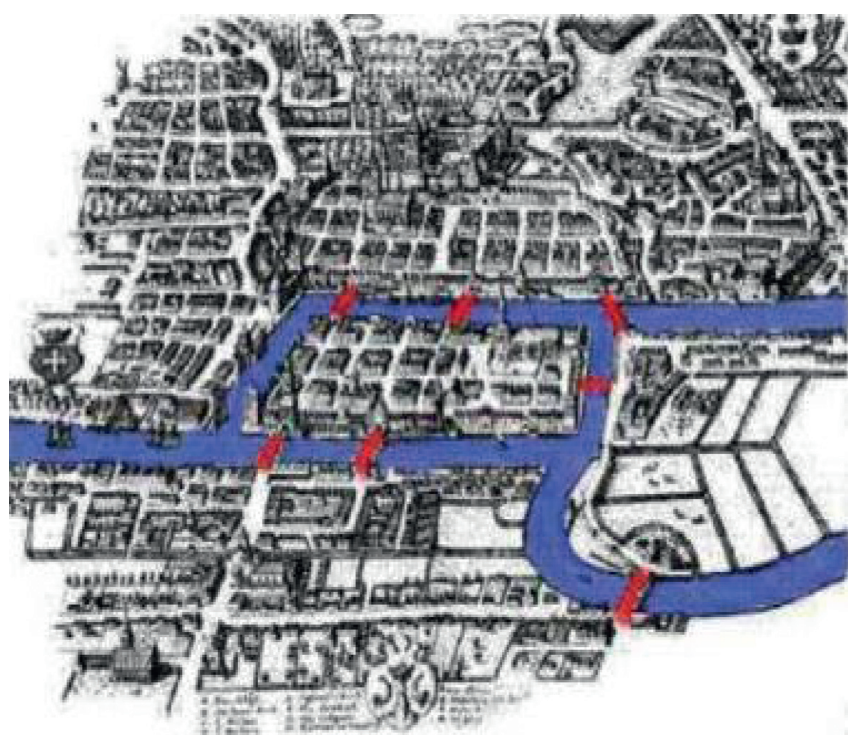

(a)

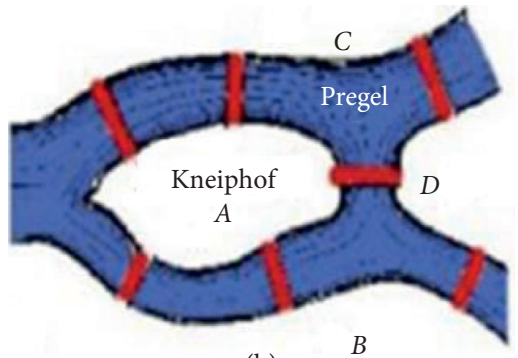

(b)

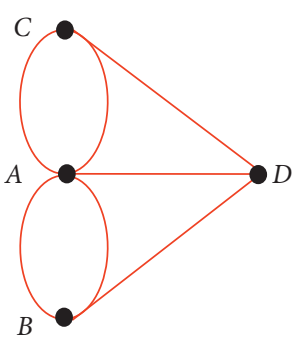

(c)

FiguRE 1: Königsberg bridge problem [9]. (a) The town of Königsberg. (b) Schematic representation of the area with the bridges. (c) Euler's representation of the problem.

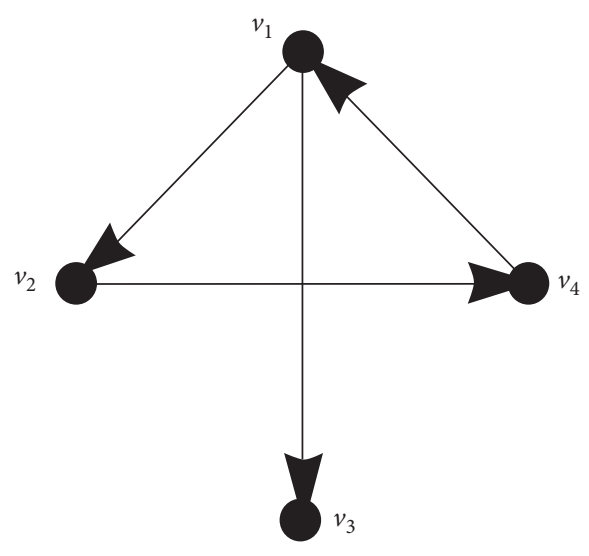

Figure 2: A graph with four vertices.

Definition 2 (see [10]). An adjacency matrix of a graph $G=$ $(V, E)$ with $n$ vertices is an $n \times n$ matrix denoted by $A=$ $\left(a_{i j}\right)$, where $a_{i j}=1$ if $E$ contains a directed edge $(j, i)$. It is an arrow pointing from vertex $j$ to vertex $i$, and $a_{i j}=0$ otherwise.

An adjacency matrix for the graph in Figure 2 is

$$
A=\left[\begin{array}{llll}
0 & 0 & 0 & 1 \\
1 & 0 & 0 & 0 \\
1 & 0 & 0 & 0 \\
0 & 1 & 0 & 0
\end{array}\right] .
$$

In 1965, Lotfi Zadeh introduced a revolutionized mathematical concept, namely. fuzzy set. A fuzzy set is a refined version of a crisp set (classical set). The membership value of an element of a crisp set is 0 or 1 . However, a fuzzy set employs membership value between 0 and 1 [11]. Formally, a fuzzy set $A$ of universe $X$ is defined by its membership function $\mu_{A}(X)$ as follows:

$$
\mu_{A}(x): X[0,1],
$$

such that $\mu_{A}(x)=1$ if $x$ is totally in $A ; \mu_{A}(x)=0$ if $x$ is not in $A$; and $0<\mu_{A}(x)<1$ if $x$ is partly in $A$.

Figure 3 shows a membership function of a fuzzy set.

These two concepts (graph and fuzzy set) have given "birth" to a new mathematical structure, namely, fuzzy graph. Definition 3 indicates that vertices and edges are both fuzzy. In other words, the vertices and edges have values between 0 and 1. Figure 4 illustrates a fuzzy graph.

Definition 3 (see [12]). A fuzzy graph $G=(\sigma, \mu)$ is a pair of functions $\sigma: S \longrightarrow[0,1]$ and $\mu: S \times S \longrightarrow[0,1] \ni \forall x, y \in S$, $\mu(x, y) \leq \sigma(x) \wedge \sigma(y)$.

An adjacency matrix of a fuzzy graph is defined as follows:

Definition 4 (see [12]). An adjacency matrix, $A$ of a fuzzy graph $G=(V, \sigma, \mu)$, is an $n \times n$ matrix defined as $A=\left(a_{i j}\right)$ such that $a_{i j}=\mu\left(v_{j}, v_{i}\right)$.

The adjacency matrix for the fuzzy graph in Figure 4 is

$$
A=\left[\begin{array}{cccc}
0 & 0 & 1 & 0.2 \\
0.4 & 0 & 0 & 0 \\
0 & 0.7 & 0 & 0 \\
0 & 0 & 0.5 & 0
\end{array}\right] .
$$




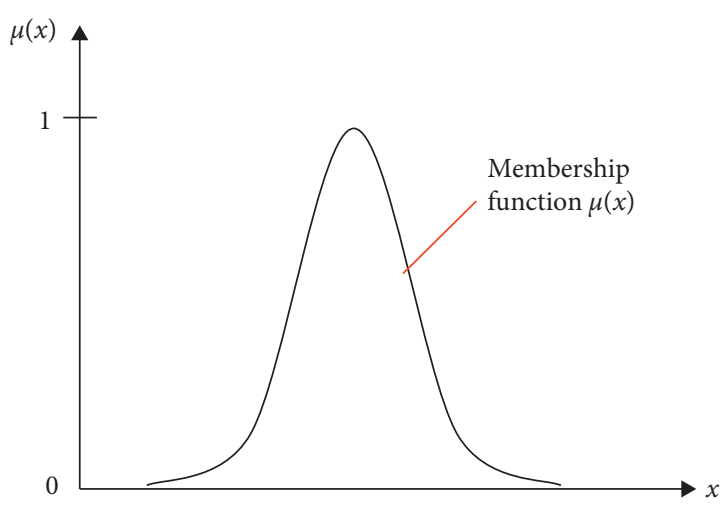

Figure 3: An example of a fuzzy set.

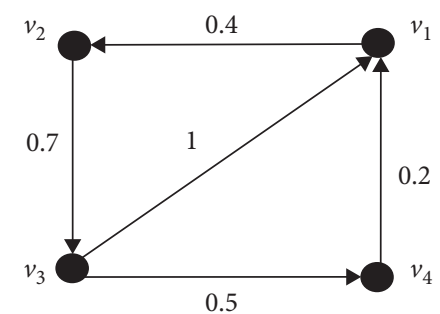

Figure 4: An example of a fuzzy graph.

Blue et al. [13] further introduced five types of fuzzy graphs, i.e., taxonomy of fuzzy graphs. They argued that fuzziness can occur in several ways, namely:

Type 1: fuzzy set of graphs

Type 2: crisp vertices and fuzzy edges

Type 3: crisp vertices and edges but with fuzzy connectivity

Type 4: fuzzy vertices and crisp edges

Type 5: crisp graph with fuzzy weight

Later, Tahir et al. [14] formalized these five types of fuzzy graphs as follows:

Type 1: $G_{F}^{1}=\left\{G_{1_{F}}, G_{2_{F}}, \ldots, G_{n_{F}}\right\}$ where fuzziness is on $G_{i_{F}}$ for $i=1,2, \ldots, n$

Type 2: $G_{F}^{2}=\left\{V, E_{F}\right\}$ where the edges are fuzzy

Type 3: $G_{F}^{3}=\left\{V, E\left(t_{F}, h_{F}\right)\right\}$ where both the vertices and edges are crisp but the edges have fuzzy heads and tails Type 4: $G_{F}^{4}=\left\{V_{F}, E\right\}$ where the vertices are fuzzy

Type 5: $G_{F}^{5}=\left\{V, E\left(w_{F}\right)\right\}$ where both the vertices and edges are crisp but the edges have fuzzy weights

In the following subsection, a brief review on a new mathematical concept called fuzzy autocatalytic set is presented.

2.1. Fuzzy Autocatalytic Set (FACS). The concept of autocatalysis was originated in chemistry, in particular, for the description of catalytically interaction between molecules
[15-17]. Further, Jain and Krishna [18] formalized the concept of an autocatalytic set (ACS) as a directed graph in which its vertices represent species and its edges represent catalytic interactions among them. The formal definition of an ACS is given as follows.

Definition 5 (see [18]). An autocatalytic set is a subgraph, each of whose vertices has at least one incoming link from vertices belonging to the same subgraph.

Some examples of ACSs are illustrated in Figure 5. The simplest ACS is a vertex with 1-cycle.

The merger of fuzzy graph and autocatalytic set has led to the idea of fuzzy autocatalytic set (FACS) by Tahir et al. [14]. The formal definition of FACS is laid as follow.

Definition 6 (see [14]). A fuzzy autocatalytic set is a subgraph each of whose vertices has at least one incoming link with membership value, $\mu\left(e_{i}\right) \in(0,1], \forall e_{i} \in E$, from any other vertices belonging to the same subgraph.

An example of FACS is given in Figure 6.

In the same paper, Tahir et al. [14] proved the following theorems regarding fuzzy graph and FACS.

Theorem 1 (see [14]). Every crisp graph is a fuzzy graph.

Theorem 2 (see [14]). Every autocatalytic set is a fuzzy graph.

Theorem 3 (see [14]). Every fuzzy autocatalytic set is also a fuzzy graph.

The relation between crisp graph, fuzzy graph, ACS, and FACS is depicted in Figure 7.

In general, graphs have been widely used to model various systems [19]. Graphs have been used in decisionmaking problems such as in industry, government, healthcare, business, and education [20]. In the next subsection, a decision-making technique called Potential Method is reviewed.

2.2. Potential Method. Potential Method is a method in decision-making process which utilizes graph. The graph is called preference. The preference graph was developed by Lavoslav Čaklović in 2002 to model pairwise comparisons between alternatives. In general, suppose $V$ is a set of alternatives in which some preferences are being considered. If an alternative $u$ is preferred over alternative $v$ (denoted as $u>v$ ), it can be presented as a directed edge from vertex $v$ to vertex $u$ (see Figure 8 ). The edge is denoted by $(u, v)$.

If the preference is described with an intensity (e.g. equal, weak, moderate, strong, or absolute preference), then the directed edge from $v$ to $u$ has a value, namely, weight, and is denoted by $F_{(u, v)}$. In case of an equal preference (denoted by $u \sim v$ ), then $F_{(u, v)}=0$, and the direction of the edge is irrelevant; i.e., edge $(u, v)$ can be replaced by $(v, u)$.

Čaklović [21] added that a preference graph has neither loops nor parallel edges. It contains at most $\left(\begin{array}{l}n \\ 2\end{array}\right)$ edges [22]. 

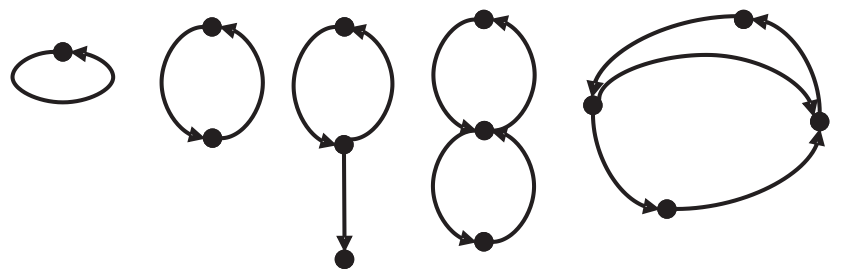

FIgURE 5: Some examples of ACS.

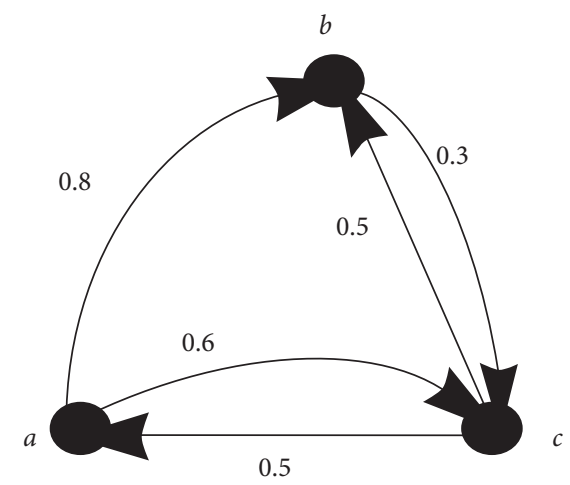

Figure 6: A FACS.

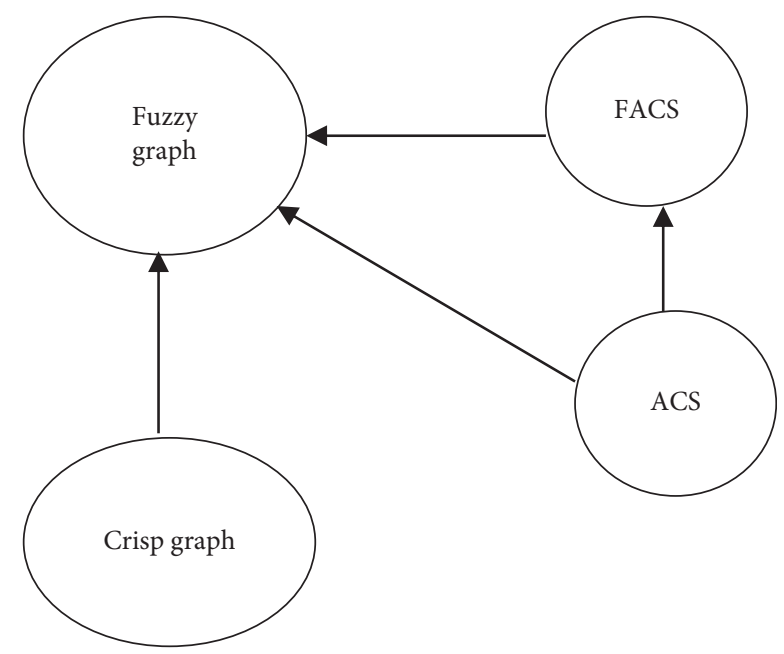

Figure 7: The relation of ACS and FACS to fuzzy graph.

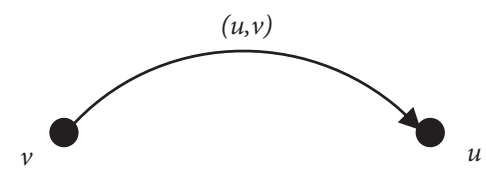

FIGURE 8: Alternative $u$ is preferred over alternative $v$.

The formal definition of a preference graph is given in Definition 7 as follows.

Definition 7 (see [23]). A preference graph is a triple $G=$ $(V, A, F)$ where $V$ is a set of $n \in \mathbb{N}$ vertices (representing alternatives), $A \subseteq V \times V$ is a set of directed edges, and $F: A \longrightarrow \mathbb{R}$ is a preference flow which maps each edge $(u, v)$ to the corresponding intensity, $F_{(u, v)}$.
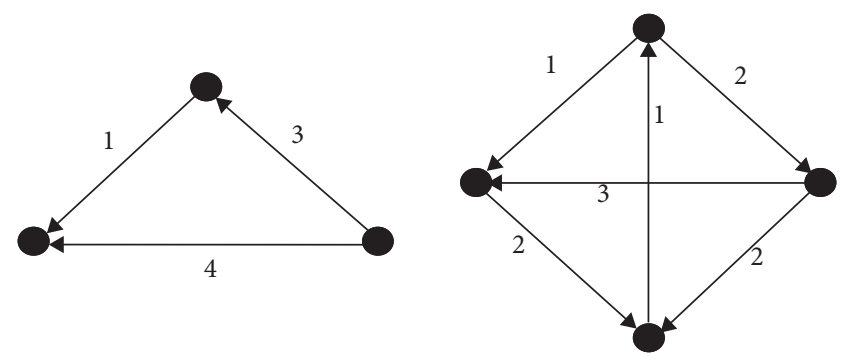

Figure 9: Examples of potential graphs.

Two examples of potential graph are illustrated in Figure 9.

Directed graphs have appeared in connection with various optimization problems, in particular, tournament problems $[24,25]$. The description of a tournament problem by means of a graph is described in the following subsection.

2.3. Tournament. Suppose that $n$ players compete in a round robin tournament whereby each player competes with all other players. In other words, for every pair of players $u$ and $v$, either $u$ beats $v$ or $v$ beats $u$ and no draw is allowed [26].

The outcome of a tournament can be represented by a directed graph $[27,28]$. Thus, for each pair of vertices $u$ and $v$ in a tournament graph, there is a directed edge from $u$ to $v$ or from $v$ to $u$, but not both. The formal definition of a tournament in form of a graph is given as follows.

Definition 8 (see [26]). A tournament graph with $n$ vertices is a pair $T=(V, E)$ where $V=\left\{v_{1}, v_{2}, \ldots, v_{n}\right\}$ is a set of vertices and $E \subset V^{2}$ is a set of ordered pairs called directed edges, so that for every two distinct vertices $v_{i}$ and $v_{j}$ either $\left(v_{i}, v_{j}\right) \in E$ or $\left(v_{j}, v_{i}\right) \in E$.

Some examples of tournament graphs are illustrated in Figure 10.

Kendall and Smith [29] introduced a procedure to compare between three objects $(A, B, C)$ that can be represented by a triangle called triad. The orientation of edges of a triad is discussed by Harary and Moser [27], Tversky [30], and Gass [31]. The transitivity for a triad is categorized as two types, simply as transitive and intransitive. They are elaborated in the following subsections.

2.3.1. Transitivity. Consider a set of objects $\{A, B, C\}$. A respondent may choose $B$ from the pair $\{A, B\}$ and $A$ from the pair $\{A, C\}$ and prefer $B$ from $\{B, C\}$. His choices can be ordered as $\{B, A, C\}$ as shown in Figure 11 . The resultant orientation is said to be a transitive [27, 29].

On the other hand, an intransitive orientation occurs when the respondent selects $B$ from the pair $\{A, B\}, A$ from the pair $\{A, C\}$, and $C$ from the pair $\{B, C\}$ as illustrated in Figure 12. An intransitive orientation produces a cycle [29].

However, only the transitive orientation is considered and discussed in this paper. 


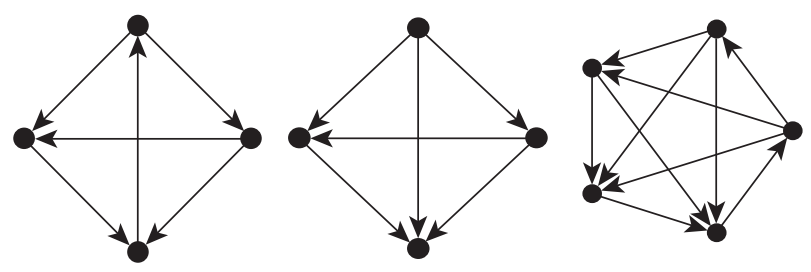

Figure 10: Examples of tournament graphs.

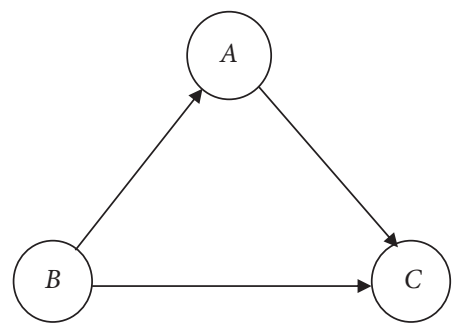

FIgURE 11: Transitive (acyclic).

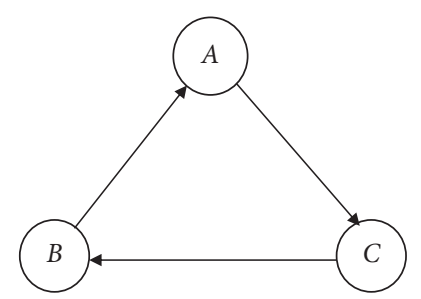

FIgURe 12: Intransitive (cyclic).

2.3.2. Transitive Tournament. An orientation of a tournament is called transitive whenever $a \longrightarrow b$ and $b \longrightarrow c$; then $a \longrightarrow c$. In other words, if $a b$ and $b c$ are edges in $T$, then $a c$ is also an edge in $T$ (see Figure 13).

A graph which contains no cycle of any length is called an acyclic graph.

Definition 9 (see [32]). A directed graph is called a directed acyclic graph if it does not contain any directed cycles.

Therefore, a transitive tournament is an acyclic graph if it contains no cycles as stated by Theorem 4 as follows.

Theorem 4 (see [27]). A tournament is transitive if and only if it contains no cycles.

An acyclic graph is categorized as weakly connected and defined by Skiena [33] and Préa [34] as follows.

Definition 10 (Skiena [33]). The nodes in a weakly connected digraph must all have either in-degree or out-degree of at least 1 .

Definition 11 (Préa [34]). The relation which defines the weak connectivity of a directed graph if there exists a path from vertex $x$ to vertex $y$ or from $y$ to $x$ and it is not transitive.

Furthermore, there are several definitions for transitive tournament as documented in Harary and Moser [27] and

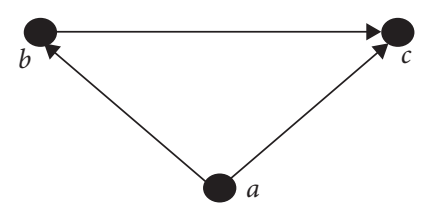

Figure 13: A transitive orientation.

Moon and Pullman [35]. However, all of them are equivalent. The following theorem lists some properties of a transitive tournament whose scores $\left(s_{1}, s_{2}, \ldots, s_{n}\right)$ are in nondecreasing order.

Theorem 5 (see [27]). Given a tournament T, the following are equivalent:

(1) $T$ is transitive

(2) $T$ contains no oriented cycle

(3) The score sequence of $T$ is $(0,1,2, \ldots, n-2, n-1)$

(4) $T$ contains $n(n-1)(n-2) / 6$ transitive triples

(5) $T$ has exactly one Hamiltonian path

A transitive tournament has exactly a sink and a source [31]. A source is a vertex with zero in-degree; i.e., ind $(v)=0$. A vertex $v$ is called a sink if all $n-1$ edges ended at $v$. A sink is a vertex with zero out-degree; i.e., $\operatorname{outd}(v)=0$. Figure 14 shows a transitive 4 -tournament. It has no cycle, i.e., acyclic. Vertices $B$ and $D$ are the source and sink, respectively.

A transitive tournament contains a source, i.e., a vertex with no incoming link. It is not an ACS by Definition 5. In other words, a transitive tournament is "not a full pledge ACS." The condition has led to a new "weak version" of ACS as described in detail in the following section.

\section{Weak Autocatalytic Set (WACS)}

This section presents the WACS. A WACS is derived from transitive tournament. It is defined formally as follows.

Definition 12. A WACS is a non-loop subgraph in which it has a vertex with no incoming link.

Figure 15 illustrates some examples of WACS.

New features of WACS are deduced. These features are inspired from the work of Skiena [33] and Préa [34] on weak connectivity. The following theorem is an immediate consequence of Definition 10 and 11.

Theorem 6. Every WACS is a weak connected graph.

Proof. Let $G=(V, E)$ be a WACS. Therefore, by Definition $12, G$ contains a vertex which has no parallel edge, say, from $u$ to $v$ or from $v$ to $u$. Every node in $G$ has either out-degree or in-degree of at least 1 . Therefore, every WACS is a weak connected digraph by Definition 10 and Definition 11. 


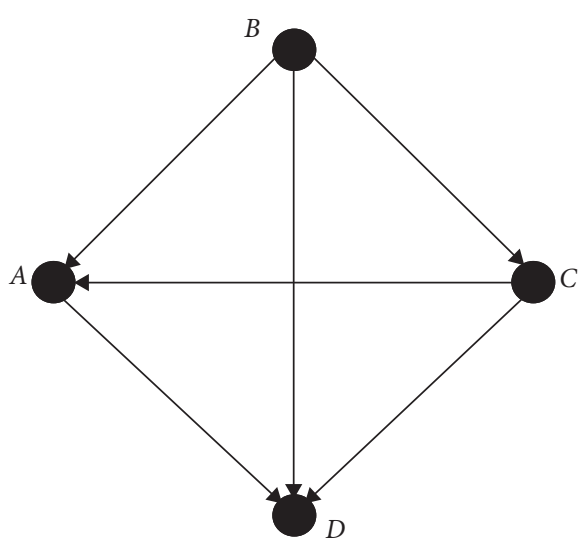

Figure 14: A transitive 4-tournament.

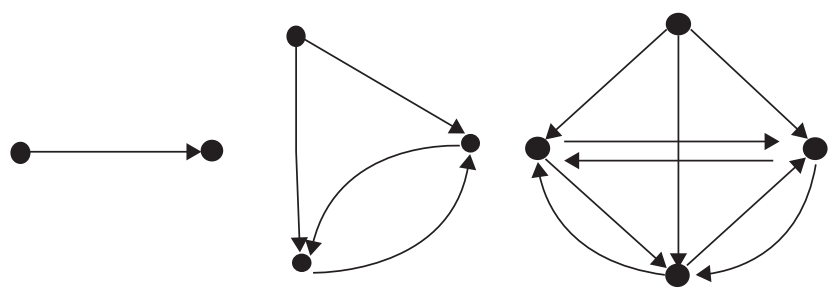

FIgURE 15: Examples of WACS.

Consequently, a WACS has no Hamiltonian cycle. This particular feature leads to the following theorem.

Theorem 7. Every WACS must have at least a path, which is not closed.

Proof. Let $G=(V, E)$ be a WACS. A closed path begins and ends at the same vertex. Since a WACS has a vertex with no incoming link, it produces an open path. Therefore, every WACS has at least a path which is not closed.

The graph in Figure 16 shows a WACS with three vertices and not closed.

A graph can be represented in form of a matrix i.e. adjacency matrix (see Definition 2). An adjacency matrix for the graph in Figure 14 is $A=\left[\begin{array}{llll}0 & 1 & 1 & 0 \\ 0 & 0 & 0 & 0 \\ 0 & 1 & 0 & 0 \\ 1 & 1 & 1 & 0\end{array}\right]$.

In a WACS, the entries of main diagonal of its adjacent matrix are all zeros. It contains a zero row, which shows that there exists a vertex with no incoming link. Hence, every WACS can be mapped to a square matrix as presented in the following theorem.

Theorem 8. Let $G(V, E)$ be a WACS defined by $G_{k}=\left\{\begin{array}{ll}0 & \text { when } i=j \text { and } e_{i} \notin E \\ \mu\left(e_{i}\right) \in\{0,1\} & \text { when } i \neq j \text { and } e_{i} \in E\end{array}\right.$ for $k=1,2, \ldots, n$. Consider $G=\left\{G_{k}: k=1,2, \ldots, n\right\}$ be a finite set of WACS and let $M_{F}^{n \times n}=\left\{\left[a_{i j}\right]^{n \times n}: a_{i j} \in\{0,1\}\right.$ with $\left.a_{i i}=0\right\}$ be a square matrix. Define $f: G \longrightarrow M_{F}^{n \times n} \ni f\left(G_{k}\right)=\left[a_{i j}\right]$. Then $f$ is a bijective function.
Proof

(i) Let $G(V, E)=G^{\prime}\left(V^{\prime}, E^{\prime}\right)$

$\Longrightarrow V=\left\{v_{1}, v_{2}, \ldots, v_{n}\right\}=\left\{v_{1}^{\prime}, v_{2}^{\prime}, \ldots, v_{n}^{\prime}\right\}=V^{\prime}$ and $E=\left\{\mu\left(v_{j}, v_{i}\right)\right\}_{i, j=1,2, \ldots, n}=\left\{\mu\left(v_{j}^{\prime}, v_{i}^{\prime}\right)\right\}_{i, j=1,2, \ldots, n}=E^{\prime}$

$\Longrightarrow \mu\left(v_{j}, v_{i}\right)=a_{i j}=a_{i j}^{\prime}=\mu\left(v_{j}^{\prime}, v_{i}^{\prime}\right)$

$\Longrightarrow\left[a_{i j}\right]=\left[a_{i j}\right]^{\prime}$

$\therefore f$ is a well - define function.

(ii) $f$ is onto since for $\left[a_{i j}\right] \in M_{F}^{n \times n}, \exists G_{k} \ni f\left(G_{k}\right)=$ $\left[a_{i j}\right]$ and $a_{i j}=\mu\left(v_{i}, v_{j}\right)$ for $\left(v_{i}, v_{j}\right) \in G_{k}$

(iii) Suppose $f\left(G_{1}(V, E)\right)=f\left(G_{2}(V, E)\right)$ $a_{i j}=a_{i j}^{\prime} \Longrightarrow\left(v_{j}, v_{i}\right)=\left(v_{j}^{\prime}, v_{i}^{\prime}\right)$

$\Longrightarrow v_{i}=v_{i}^{\prime}$ and $v_{j}=v_{j}^{\prime}, \forall i, j=1,2, \ldots, n$

$\Longrightarrow G_{1}(V, E)=G_{2}(V, E)$.

$\therefore f$ is one to one

In general, a WACS with $n$ vertices contains a vertex with no multiple edges. Consequently, the vertex will have $n-1$ edges. However, the other $n-1$ vertices may have multiple edges. The following theorem proves that a finite set of vertices for WACS produces a finite set of edges.

Theorem 9. If $G(V, E)$ is a WACS and $|V|=n$, then $|E| \leq(n-1)^{2}$.

Proof. Let $G(V, E)$ be a WACS and $V=\left\{v_{1}, v_{2}, v_{3}, \ldots, v_{n}\right\}$. There exist a vertex with no incoming link and $|V|=n$. The most possible edges for $G$ is 


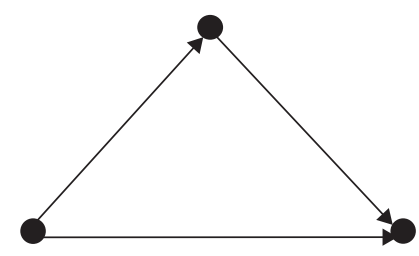

FIgURE 16: A WACS with path, which is not closed.

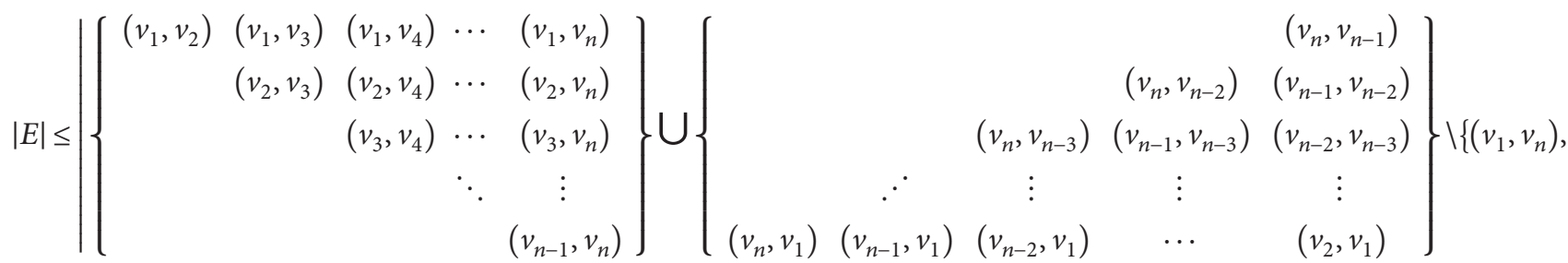

$\left.\cdot\left(v_{2}, v_{n}\right),\left(v_{3}, v_{n}\right), \ldots,\left(v_{n-1}, v_{n}\right)\right\} \mid$

Hence,

$$
\begin{aligned}
|E(n)| \leq & {\left[\sum_{j=2}^{n}\left|\left(v_{1}, v_{j}\right)\right|+\sum_{j=3}^{n}\left|\left(v_{2}, v_{j}\right)\right|+\sum_{j=4}^{n}\left|\left(v_{3}, v_{j}\right)\right|+\cdots+2+1\right]+} \\
& \cdot\left[1+2+\cdots+\sum_{j=4}^{n}\left|\left(v_{j}, v_{3}\right)\right|+\sum_{j=3}^{n}\left|\left(v_{j}, v_{2}\right)\right|+\sum_{j=2}^{n}\left|\left(v_{j}, v_{1}\right)\right|\right]-\left[\sum_{i=1}^{n-1}\left|\left(v_{i}, v_{n}\right)\right|\right] .
\end{aligned}
$$

Consequently,

$$
\begin{aligned}
|E| & \leq\left(\begin{array}{l}
n \\
2
\end{array}\right)+\left(\begin{array}{l}
n \\
2
\end{array}\right)-(n-1) \\
& =2\left(\begin{array}{l}
n \\
2
\end{array}\right)-(n-1) \\
& =2\left(\frac{n !}{2 !(n-2) !}\right)-(n-1) \\
& =\frac{n !}{(n-2) !}-(n-1) \\
& =\frac{n(n-1)(n-2) !}{(n-2) !}-(n-1) \\
& =n^{2}-2 n+1 \\
& =(n-1)(n-1) \\
& =(n-1)^{2} .
\end{aligned}
$$

Therefore, $|E| \leq(n-1)^{2}$

Hence, a WACS with $n$ vertices has number of edges at most $(n-1)^{2}$.
The following subsection establishes the relation between transitive tournament and WACS.

3.1. Transitive Tournament as a WACS. A tournament consists of pairwise comparisons between objects. The procedure for the comparisons is discussed in Kendall [24] and David [25]. The most possible number of edges for a tournament graph is formalized in Theorem 10 as follows.

Theorem 10. The most possible edges for a tournament graph $T(V, E)$ with $n$ vertices is $\left(\begin{array}{l}n \\ 2\end{array}\right)$.

Proof. Suppose $T(V, E)$ is a tournament graph with $n$ vertices.

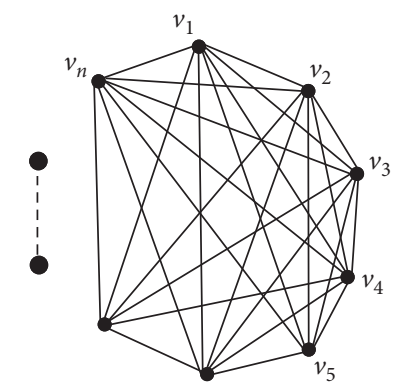

All its possible edges can be written as a set $E$ such that 


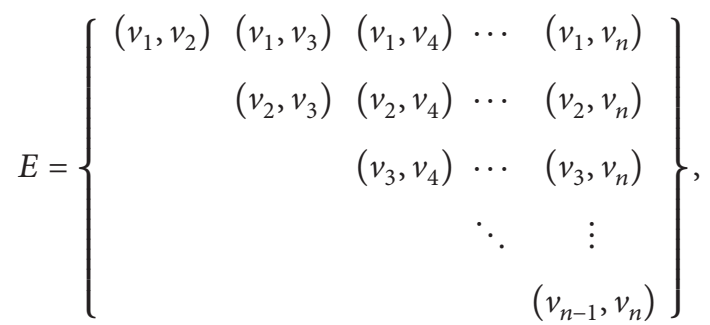

and the number of edges of $E$ is

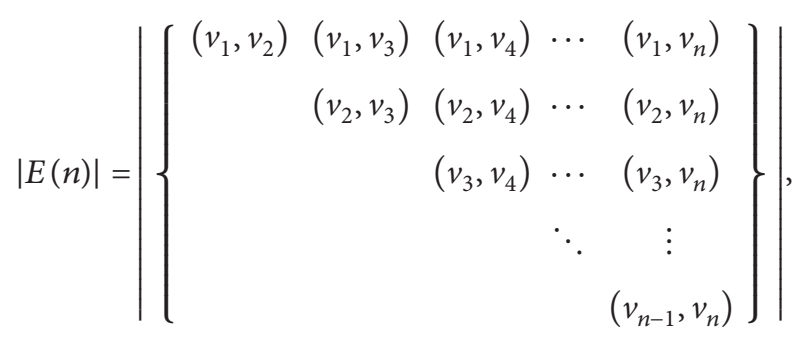

$$
\begin{aligned}
& |E(n)| \leq \sum_{j=2}^{n}\left|\left(v_{1}, v_{j}\right)\right|+\sum_{j=3}^{n}\left|\left(v_{2}, v_{j}\right)\right|+\sum_{j=4}^{n}\left|\left(v_{3}, v_{j}\right)\right|+\cdots+2+1 \text {. }
\end{aligned}
$$

In particular,

$$
\begin{aligned}
|E(n)|= & (n-1)+(n-2)+\cdots+2+1 \\
2|E(n)|= & {[(n-1)+(n-2)+\cdots+2+1]+[(n-1)+(n-2)} \\
& +\cdots+2+1] \\
= & {[(n-1)+(n-2)+\cdots+2+1]+[1+2+\cdots} \\
& +(n-2)+(n-1)] \\
= & n+n+n+\cdots+n \\
= & n(n-1) .
\end{aligned}
$$

Thus, $|E(n)|=n(n-1) / 2=\left(\begin{array}{l}n \\ 2\end{array}\right)$.

Such that $\left(v_{i}, v_{j}\right)$ means $\left(v_{i}, v_{j}\right)$ or $\left(v_{j}, v_{i}\right)$.

Hence, the most possible number of edges for $T(V, E)$ is $\left(\begin{array}{l}n \\ 2\end{array}\right)$.

A comparison number of edges between WACS and transitive tournament is established, namely the number of edges produced by a tournament is less than the number of edges produced by a WACS with respect to a given graph. The following lemma is needed.

Lemma 1. $\left(\begin{array}{l}n \\ 2\end{array}\right)<(n-1)^{2}$ for $n>1$.
Proof.

$$
\begin{aligned}
\left(\begin{array}{l}
n \\
2
\end{array}\right) & =\frac{n !}{2 !(n-2) !} \\
& =\frac{n(n-1)(n-2) !}{2 !(n-2) !} \\
& =\frac{n(n-1)}{2 !}=\frac{n(n-1)}{2} \\
& =\frac{n}{2}(n-1) \\
& <(n-1)(n-1) \text { for } n>1 \\
\text { since } \frac{n}{2} & =\frac{n-1+1}{2}=\frac{n-1}{2}+\frac{1}{2}<n-1+\frac{1}{2} \text { whenever } n>1 \\
& =(n-1)^{2}
\end{aligned}
$$

Therefore, $\left(\begin{array}{l}n \\ 2\end{array}\right)<(n-1)^{2}$.

Consequently, the relation between a transitive tournament and a WACS is established by the following theorem.

Theorem 11. Every transitive tournament is a WACS.

Proof. Firstly, a transitive tournament is guaranteed to have a vertex with no incoming link by Theorem 4 as well as no loops by Theorem 5. Furthermore, it contains $\left(\begin{array}{l}n \\ 2\end{array}\right)$ number of edges. Theorem 9 and Theorem 10 guarantee that the possible maximum number of edges produced by a WACS is $(n-1)^{2}$ for the same number of $n$ vertices. Therefore, the number of edges for a transitive tournament will not exceed the number of edges of a WACS by Lemma 1 . Thus, every transitive tournament is a WACS by Definition 12 .

Figure 17 depicts the relation between transitive tournament and ACS.

The aim of this paper is to establish the relation between ACS and PM. In the following section, the preference graph of Potential Method (PM) is reviewed. In this work, we use the term "potential graph" in short to refer the preference graph of the Potential Method. A brief definition of PM are presented in Section 2.2. There are two types of orientation as pointed in Section 2.3.1. In this paper, we consider the transitive orientation for the potential graph.

\section{Transitive Potential Graph}

The following example is obtained from Čaklović and Kurdija [23] with some additional information to illustrate a transitive orientation for the potential graph. 


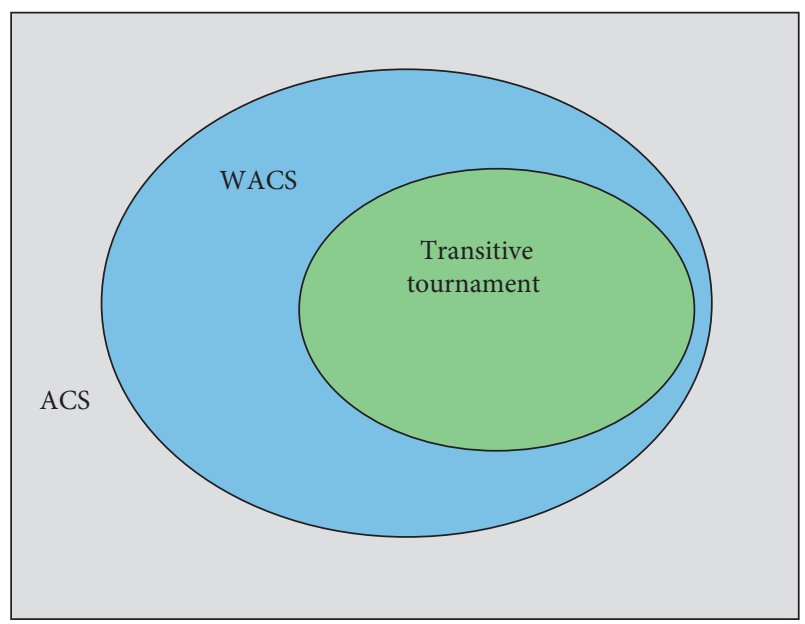

FIgURE 17: Transitive tournament is a WACS.

Example 1. Let $a, b, c$ and $d$ be preference items. Assume the following preferences over $a, b, c$, and $d$.

(i) $a$ is preferred to $b$ by 1 .

(ii) $a$ is better than $c$ by 2 .

(iii) $a$ is preferred to $d$ by 4 .

(iv) $b$ is better than $c$ by 1 .

(v) $b$ is better than $d$ by 3 .

(vi) $c$ is more preferred to $d$ by 2

In short, the preferences are $a>b, a>c, a>d, b>c$, $b>d$, and $c>d$ with intensities of $1,2,4,1,3$, and 2, respectively. The following are the edges of the preferences:

(i) Edge $(a, b)$ with flow $F_{(a, b)}=1$

(ii) Edge $(a, c)$ with flow $F_{(a, c)}=2$

(iii) Edge $(a, d)$ with flow $F_{(a, d)}=4$

(iv) Edge $(b, c)$ with flow $F_{(b, c)}=1$

(v) Edge $(b, d)$ with flow $F_{(b, d)}=3$

(vi) Edge $(c, d)$ with flow $F_{(c, d)}=2$

The transitive potential graph is illustrated in Figure 18. The vertices represent the preference items $a, b, c$, and $d$. The intensity value for an edge represents the strength between two preference items.

Clearly, any transitive potential graph contains no cycle of any length. This situation leads to the following theorem.

Theorem 12. A potential graph is transitive if and only it contains no cycles.

Proof. $(\Longrightarrow)$ Assume a potential graph $G$ is transitive but contains a cycle $v_{1}, v_{2}, \ldots, v_{n}, v_{1}$. For $n=2, G$ contains parallel arcs $v_{1} v_{2}$ and $v_{2} v_{1}$, thus contradicting $G$ being a potential graph. Further, for $n \geq 3, v_{1} v_{2}$ and $v_{2} v_{3}$ are arcs; transitivity implies $v_{1} v_{3}$ is an arc. Now we know that $v_{1} v_{3}$ is an arc, and since $v_{3} v_{4}$ is an arc, again transitivity implies $v_{1} v_{4}$ is an arc. We reason like this, $n-2$ times to conclude that $v_{1} v_{k}$ is an arc. However, $v_{k} v_{1}$ is also an arc of the digraph since it is part of the cycle. Thus, $G$ contains parallel $\operatorname{arcs} v_{1} v_{k}$ and $v_{k} v_{1}$, contradicting $G$ being a potential graph.

$(\Longleftarrow)$ Assume a potential graph $G$ contains no cycle. Suppose $G$ contains arcs $u v$ and $v w$. We know $u \neq w$ since $G$ contains no parallel arcs. The potential graph cannot have $w u$ as an arc because if it did, it would contain the cycle $u, v, w, u$. Since it is a potential graph and $u \neq w$, either $u w$ or $w u$ is an arc. Thus, $u w$ is an arc. Hence, $G$ is transitive.

Some examples of transitive potential graphs are shown in Figure 19.

Thus, a transitive potential graph is indeed a WACS and presented in the following theorem.

Theorem 13. Every transitive potential graph is a WACS.

Proof. A transitive potential graph has neither loops nor parallel edges. It is a weak connected graph by Theorem 6 . Hence, it contains no cycle of any length as indicated by Theorem 12. Thus, it produces an open path by Theorem 7 . Therefore, by Definition 12 every transitive potential graph is a WACS.

Potential graph usually arises from optimization or multicriteria problem. Hence, such transformed problem can be studied as a special graph, namely, WACS, rigorously. A WACS is a crisp graph since its edges are either 0 or 1 . The following section discusses the relation of WACS and fuzzy graph.

4.1. Weak Autocatalytic Set as Fuzzy Graph. The concept of fuzzy graph is the fuzzification of the crisp graph using fuzzy set. A fuzzy graph is a replication of a crisp graph [36]. Theorem 14 proves that WACS is a special case of a fuzzy graph.

\section{Theorem 14. Every WACS is a fuzzy graph.}

Proof. Every crisp graph is a fuzzy graph by Theorem 1 . Therefore, every WACS is also a fuzzy graph since WACS is just a special kind of a crisp graph.

A WACS is a crisp graph. All crisp graphs are fuzzy but not the other way around. Therefore the converse of the above theorem is not true.

The following theorem is an immediate consequence of Theorem 14.

Theorem 15. Every transitive potential graph is a fuzzy graph.

Proof. Every transitive potential graph is a WACS by Theorem 13. Hence, every WACS is a fuzzy graph by Theorem 14. Consequently, every transitive potential graph is a fuzzy graph.

The converse of Theorem 15 is not true by similar argument as Theorem 14 earlier; i.e., all crisp graphs are fuzzy but not the other way around. 


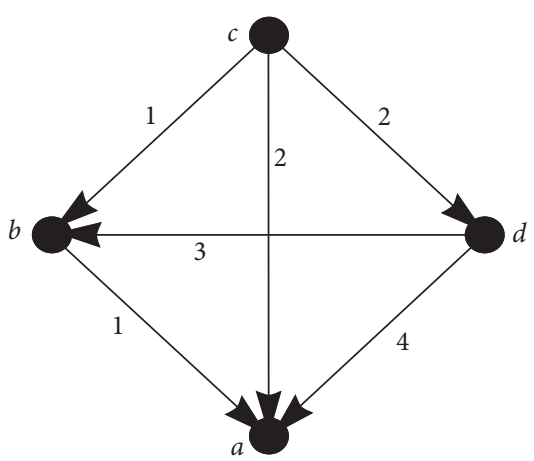

Figure 18: Transitive potential graph.

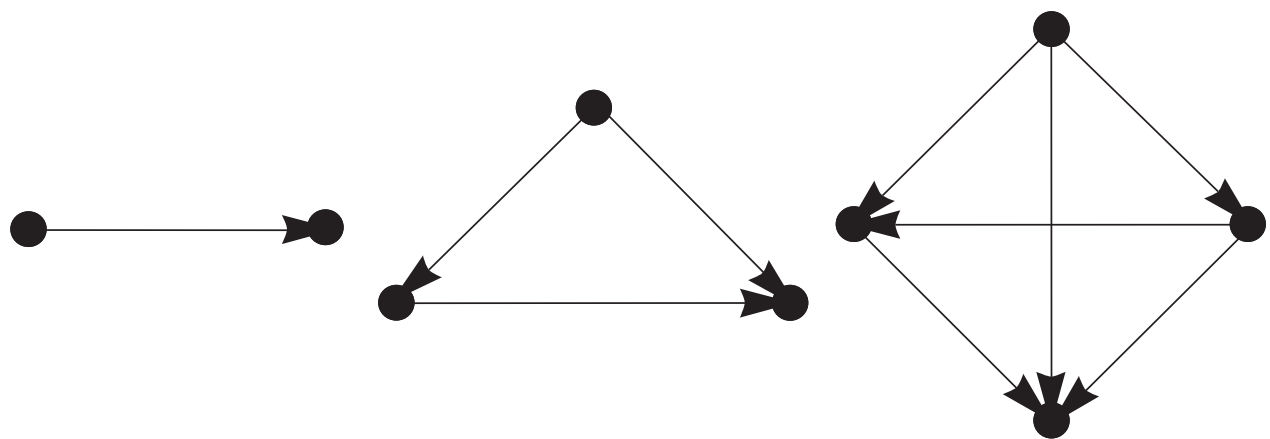

FIGURE 19: Examples of transitive potential graphs.

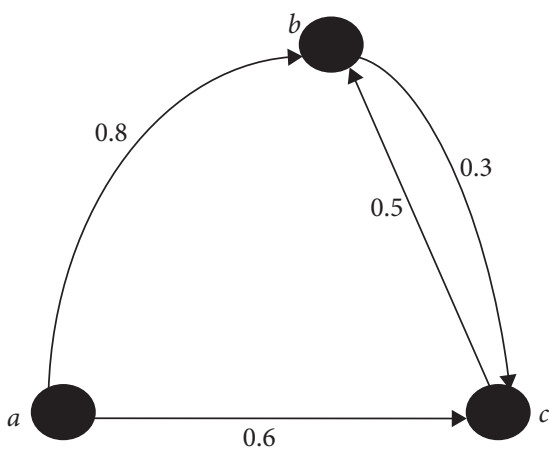

Figure 20: A FWACS.

In the following section, a new concept called fuzzy weak autocatalytic set is outlined.

\section{Fuzzy Weak Autocatalytic Set}

The fuzzification of WACS has led to a new structure, namely, fuzzy weak autocatalytic set (FWACS). The definition of FWACS is formalized in Definition 13. An example of a FWACS is shown in Figure 20.

Definition 13. A fuzzy weak autocatalytic set (FWACS) is a WACS such that every edge $e_{i}$ has a membership value, $\mu\left(e_{i}\right) \in[0,1]$ for $e_{i} \in E$.

A WACS is a crisp graph and it is a special type of FWACS as presented in Theorem 16.
Theorem 16. Every WACS is a FWACS.

Proof. Every crisp graph is a fuzzy graph by Theorem 1 . Thus, every ACS is a special case of FACS; hence every WACS is FWACS.

Furthermore, the following theorem is an immediate consequence of Theorem 16.

Theorem 17. Every FWACS is a fuzzy graph.

Proof. Theorem 1 established that every crisp graph is a fuzzy graph. Every FACS is also a fuzzy graph by Theorem 3, which immediately implies every FWACS is fuzzy graph.

There are 5 types of crisp graph as documented in Blue et al. [13]. Every crisp graph is a fuzzy graph and the converse is not true. Not all crisp graphs are autocatalytic sets. Clearly, the converse of Theorem 17 is not true either.

We have proven that a transitive potential graph is a WACS and a special case of fuzzy graph. The next section introduces preferences flow on edges.

5.1. Fuzzy Edges of Transitive Potential Graph. Consider the potential graph in Figure 18. The adjacency matrix for the graph is 


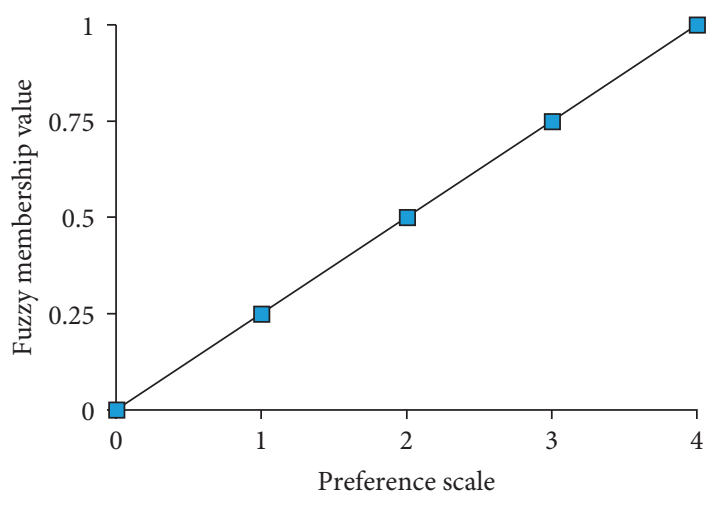

FIGURE 21: Linear transformation of preference flows.

TAвLE 1: Fuzzy membership value of preference scale.

\begin{tabular}{lcc}
\hline Preference scale & Intensity preference of potential method & Fuzzy number \\
\hline Equal & 0 & 0 \\
Weak & 1 & 0.25 \\
Fairly strong & 2 & 0.5 \\
Very strong & 3 & 0.75 \\
Absolute & 4 & 1 \\
\hline
\end{tabular}

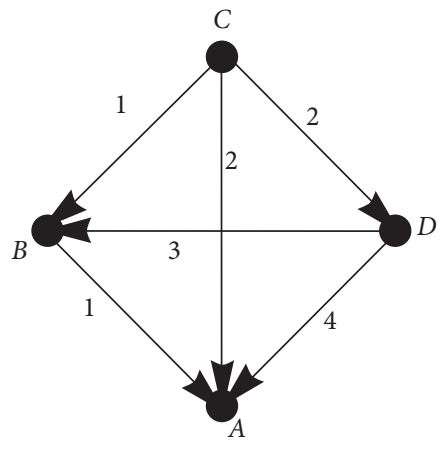

(a)

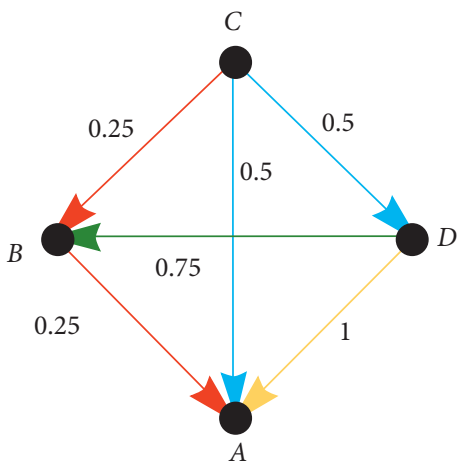

(b)

Figure 22: Crisp and fuzzy graphs of Example 1. (a) Transitive potential graph. (b) FWACS.

$$
A=\left[\begin{array}{llll}
0 & 1 & 1 & 1 \\
0 & 0 & 1 & 1 \\
0 & 0 & 0 & 0 \\
0 & 0 & 1 & 0
\end{array}\right]
$$

The transformation of the preference flows to fuzzy membership values is described in the next subsection.

5.1.1. Membership Value of Fuzzy Edge via Linear Transformation. The preference flows interval of Example 1 is transformed to fuzzy membership values linearly as illustrated in Figure 21.
In Example 1, the interval $[0,4]$ is used to indicate the strength of alternatives. Table 1 lists the transformed intensity preference to fuzzy membership values.

The crisp and fuzzy graphs of Example 1 are illustrated in Figure 22.

The same color and thickness of each edge in the crisp graph (see Figure 22(a)) indicate that the connectivity among vertices is considered the same. The value 1 is assigned when there is an edge between two vertices. If there is no edge, the value 0 is given.

Figure 22(b) illustrates the fuzzy graph. The edges have different "strengths" which are determined by their membership values. Furthermore, the greater the membership value, the stronger the connection between two vertices of 


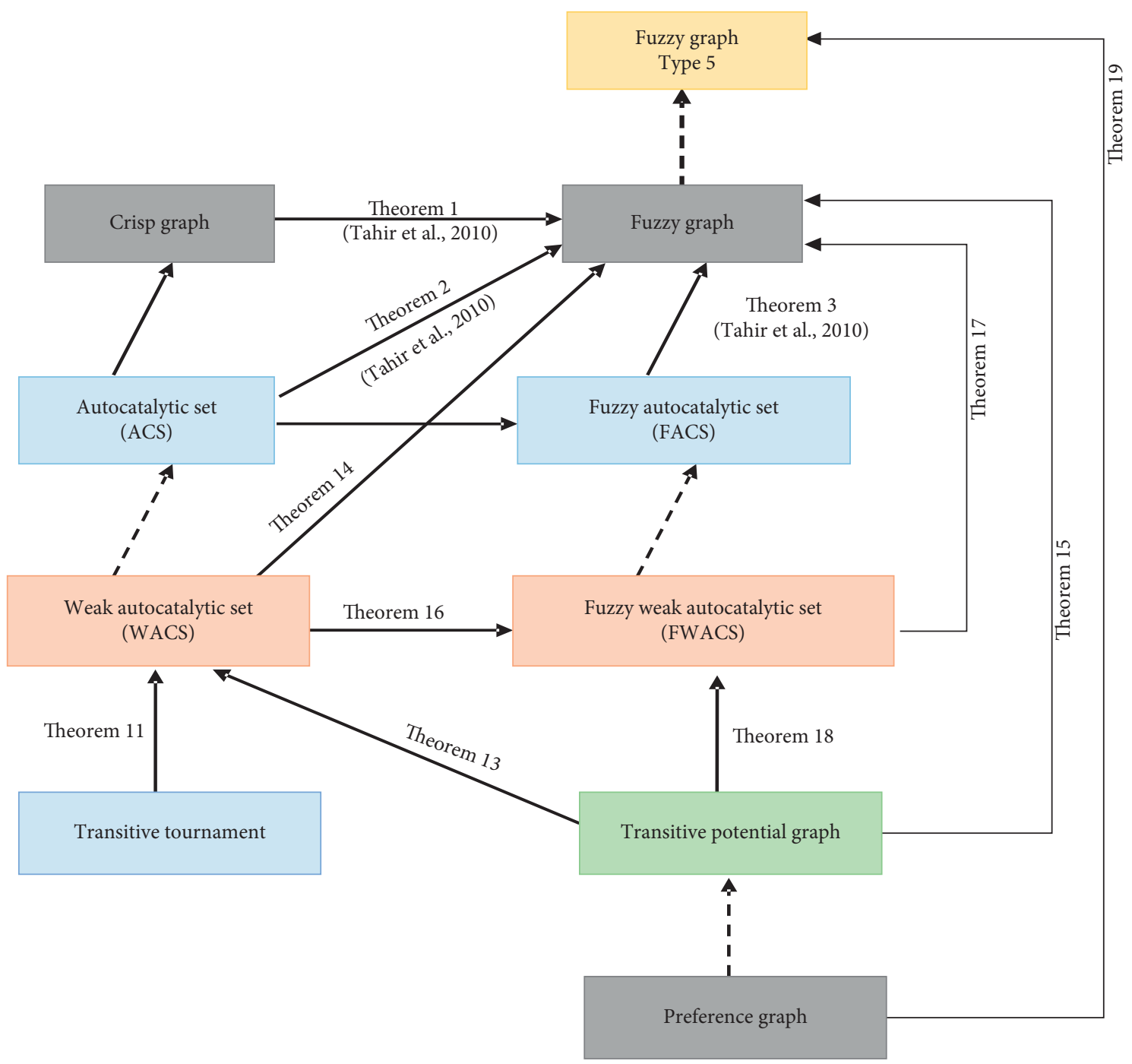

Figure 23: A schematic diagram to illustrate the relation between preference and fuzzy graphs.

the graph. Hence, different thickness and color of an edge represent the strength connection of its vertices.

Thus, the adjacency matrix for the FWACS is

$$
A=\left[\begin{array}{cccc}
0 & 0.25 & 0.5 & 1 \\
0 & 0 & 0.25 & 0.75 \\
0 & 0 & 0 & 0 \\
0 & 0 & 0.5 & 0
\end{array}\right] .
$$

In the following subsection, we present the relation between transitive potential graph and FWACS.

5.2. Transitive Potential Graph as FWACS. The relation between transitive potential graph and FWACS can be observed immediately. The relation is formalized in the following theorem.

Theorem 18. Every transitive potential graph is a FWACS.

Proof. Theorem 13 proves that a transitive potential graph is a WACS. A transitive potential graph is a fuzzy graph by
Theorem 14. The edges are fuzzy with membership values of $[0,1]$. Therefore, every transitive potential graph is a FWACS by Definition 13.

Every FWACS is a fuzzy graph by Theorem 17. But the converse of Theorem 15 is not true; i.e. a fuzzy graph is not necessarily a transitive potential graph. Consequently, the converse of Theorem 18 is not true either.

5.3. Preference Graph as Fuzzy Graph Type 5. As a matter of fact, the general version of Theorem 18 can be deduced directly from the recent definition of preference graph (see Definition 7) introduced by Čaklović and Kurdija [23].

Theorem 19. Every preference graph is a fuzzy graph Type 5 .

Proof. Let $G(V, A, F)$ be a preference graph as defined by Definition 7. Consider the preference flow $F: A \longrightarrow \mathbb{R}$ as given in Definition 7 . The $\mathbb{R}$ is homeomorphic to $(0,1)$, i.e. $\mathbb{R} \cong(0,1)[37]$. But then, $(0,1) \subset[0,1]$; hence the preference flow $F$ can be written as $F: A \longrightarrow(0,1) \subset[0,1]$ 
since $\mathbb{R} \cong(0,1)$. Consequently, $G(V, A, F)$ is now a fuzzy graph Type 5 as categorized by Blue et al. [13] and Tahir et al. [14].

A fuzzy graph Type 5 is a crisp graph with fuzzy weights for its edges defined in Blue et al. [13]. On the other hand, a preference graph is a triple $G=(V, A, F)$ where $V$ is a set of $n \in \mathbb{N}$ vertices (representing alternatives), $A \subseteq V \times V$ is a set of directed edges, and $F: A \longrightarrow \mathbb{R}$ is a preference flow which maps each edge $(u, v)$ to the corresponding intensity, $F_{(u, v)}$, as stated in Definition 7. However, fuzzy graph Type 5 is more general than preference graph since the former can accommodate directed or undirected edges. Hence, it is quite obvious that every preference graph is a fuzzy graph Type 5 and not the converse.

Figure 23 summarizes the relation between ACS, WACS, FACS, and FWACS.

\section{Conclusion}

This paper has introduced a novel definition of weak version of ACS, namely, WACS. The WACS is originally derived from transitive tournament which serves as a "bridge" between PM and ACS. The WACS has offered some new insights into PM. Furthermore, the FWACS is defined for PM with uncertainty where the edges are fuzzy. Finally, we proved that any preference graph of PM is a fuzzy graph Type 5.

In short, the major thrust of this paper is to materialize the framework proposed by Čaklović $[21,22]$ for PM. The developed FWACS is able to handle multicriteria decision making (MCDM) of uncertainty problems. Some of them can be found in Siti Salwana et al. [38].

\section{Data Availability}

The data obtained from Caklovic and Kurdija [23] [https:// doi.org/10.1016/j.ejor.2016.10.032] with some additional information have been used to support our findings on transitive potential graph and its relation to fuzzy graph.

\section{Conflicts of Interest}

The authors declare that there are no conflicts of interest regarding the publication of this paper.

\section{Acknowledgments}

This work was supported by Fundamental Research Grant Scheme (FRGS) vote 4F756 from Ministry of High Education (MOHE), Malaysia, and MyBrainSc Scholarship.

\section{References}

[1] R. V. Rao, "A decision-making framework model for evaluating flexible manufacturing systems using digraph and matrix methods," The International Journal of Advanced Manufacturing Technology, vol. 30, no. 11-12, pp. 1101-1110, 2006.
[2] A. Dey, "Vertex coloring of a fuzzy graph using alpa cut," International Journal of Managment, IT and Engineering, vol. 2, no. 8, pp. 340-352, 2012.

[3] A. Dey, L. Son, P. Kumar, G. Selvachandran, and S. Quek, "New concepts on vertex and edge coloring of simple vague graphs," Symmetry, vol. 10, no. 9, p. 373, 2018.

[4] A. Dey and A. Pal, "Fuzzy graph coloring technique to classify the accidental zone of a traffic control," Annals of Pure and Applied Mathematics, vol. 3, no. 2, pp. 169-178, 2013.

[5] A. Dey, R. Pradhan, A. Pal, and T. Pal, "The fuzzy robust graph coloring problem," in Proceedings of the 3rd International Conference on Frontiers of Intelligent Computing: Theory and Applications (FICTA) 2014, pp. 805-813, Springer, Cham, Switzerland, 2015.

[6] A. Dey, A. Pal, and T. Pal, "Interval type 2 fuzzy set in fuzzy shortest path problem," Mathematics, vol. 4, no. 4, p. 62, 2016.

[7] A. Dey, R. Pradhan, A. Pal, and T. Pal, "A genetic algorithm for solving fuzzy shortest path problems with interval type-2 fuzzy arc lengths," Malaysian Journal of Computer Science, vol. 31, no. 4, pp. 255-270, 2018.

[8] T. Wuest, B. Knoke, and K.-D. Thoben, "Applying graph theory and the product state concept in manufacturing," Procedia Technology, vol. 15, pp. 349-358, 2014.

[9] L. A. N. Amaral and J. M. Ottino, "Complex systems and networks: challenges and opportunities for chemical and biological engineers," Chemical Engineering Science, vol. 59, no. 8-9, pp. 1653-1666, 2004.

[10] F. Harary, Graph Theory, Addison Wesley Publishing Company, Boston, MA, USA, 1969.

[11] A. Dey, A. Pal, and H. V. Long, "Fuzzy minimum spanning tree with interval type 2 fuzzy arc length: formulation and a new genetic algorithm," Soft Computing, vol. 24, pp. 3963$3974,2010$.

[12] A. Rosenfeld, "FUZZY GRAPHS: the support of the office of computing activities, national science foundation, under Grant GJ-32258X, is gratefully acknowledged, as is the help of shelly rowe in preparing this paper," Fuzzy Sets and their Applications to Cognitive and Decision Processes, vol. 513, pp. 77-95, 1975.

[13] M. Blue, B. Bush, and J. Puckett, "Unified approach to fuzzy graph problems," Fuzzy Sets and Systems, vol. 125, no. 3, pp. 355-368, 2002.

[14] A. Tahir, B. Sabariah, and A. Khairil Anuar, "Modeling a clinical incineration process using fuzzy autocatalytic set," Journal of Mathematical Chemistry, vol. 47, no. 4, pp. 12631273, 2010.

[15] S. A. Kauffman, "Cellular homeostasis, epigenesis and replication in randomly aggregated macromolecular systems," Journal of Cybernetics, vol. 1, no. 1, pp. 71-96, 1971.

[16] O. E. Rössler, "Ein systemtheoretisches modell zur biogenese/ a system theoretic model of biogenesis," Zeitschrift für Naturforschung B, vol. 26, no. 8, pp. 741-746, 1971.

[17] M. Eigen, "Selforganization of matter and the evolution of biological macromolecules," Die Naturwissenschaften, vol. 58, no. 10, pp. 465-523, 1971.

[18] S. Jain and S. Krishna, "Autocatalytic sets and the growth of complexity in an evolutionary model," Physical Review Letters, vol. 81, no. 25, pp. 5684-5687, 1998.

[19] S. G. Shirinivas, S. Vetrivel, and N. M. Elango, "Applications of graph theory in computer science an overview," International Journal of Engineering Science and Technology, vol. 2, no. 9, pp. 4610-4621, 2010.

[20] R. V. Rao, Decision Making in the Manufacturing Environment: Using Graph Theory and Fuzzy Multiple Attribute 
Decision Making Methods, Springer Science \& Business Media, Berlin, Germany, 2007.

[21] L. Čaklović, "Interaction of criteria in grading process," in Proceedings of the Knowledge Society-Challenges to Management Globalization Regionalism and EU Enlargement, Koper, Slovenia, 2004.

[22] Čaklović, L., Decision making via potential method. preprint, 2002.

[23] L. Čaklović and A. S. Kurdija, "A universal voting system based on the Potential Method," European Journal of Operational Research, vol. 259, no. 2, pp. 677-688, 2017.

[24] M. G. Kendall, "Further contributions to the theory of paired comparisons," Biometrics, vol. 11, no. 1, pp. 43-62, 1955.

[25] H. A. David, The Method of Paired Comparisons, Vol. 12, Griffin, London, 1963.

[26] H. A. David, "Tournaments and paired comparisons," Biometrika, vol. 46, no. 1/2, pp. 139-149, 1959.

[27] F. Harary and L. Moser, "The theory of round robin tournaments," The American Mathematical Monthly, vol. 73, no. 3, pp. 231-246, 1966.

[28] J. W. Moon and N. J. Pullman, "On the powers of tournament matrices," Journal of Combinatorial Theory, vol. 3, no. 1, pp. 1-9, 1967.

[29] M. G. Kendall and B. B. Smith, "On the method of paired comparison," Biometrika, vol. 31, no. 3-4, pp. 324-345, 1940.

[30] A. Tversky, "Intransitivity of preferences," Psychological Review, vol. 76, no. 1, pp. 31-48, 1969.

[31] S. I. Gass, "Tournaments, transitivity and pairwise comparison matrices," The Journal of the Operational Research Society, vol. 49, no. 6, pp. 616-624, 1998.

[32] M. N. Swamy and K. Thulasiraman, Graphs: Theory and Algorithms, John Wiley \& Sons, Hoboken, NJ, USA, 1992.

[33] S. Skiena, Implementing Discrete Mathematics: Combinatorics and Graph Theory with Mathematica, Addison-Wesley, Boston, MA, USA, 1990.

[34] P. Préa, "About weak connectivity," Discrete Mathematics, vol. 165-166, pp. 579-586, 1997.

[35] J. W. Moon and N. J. Pullman, "On generalized tournament matrices," SIAM Review, vol. 12, no. 3, pp. 384-399, 1970.

[36] B. Sabariah, A. Tahir, and M. Y. Mohd Rashid, "Relationship between fuzzy edge connectivity and the variables in clinical waste incineration process," Matematika, vol. 25, pp. 31-38, 2009.

[37] F. S. Cater, "On order topologies and the real line. real analysis exchange,” 1999, https://projecteuclid.org/euclid.rae/1230995411.

[38] M. Siti Salwana, A. Tahir, A. Siti Rahmah, and M. Muhammad Zilullah, "Ranking by fuzzy weak autocatalytic set," in Proceedings of the International Conference on Soft Computing in Data Science, pp. 161-172, Bangkok, Thailand, 2018. 